\title{
Korruption in Entwicklungsländern: Abbau durch Demokratieaufbau? Eine empirische Analyse
}

\author{
Von Nadja Memmer, Brisbane*
}

Nach Berichten der Vereinten Nationen leben heute weltweit 1,2 Milliarden Menschen mit weniger als einem Dollar am Tag. ${ }^{1}$ Eine Bilanz, die nach Jahrzehnten enormer Bemühungen der Internationalen Staatengemeinschaft, Armut in Entwicklungsländern (EL) zu bekämpfen, sehr ernüchtert. Korruption - klassisch definiert als ,the misuse of public power for private profit ${ }^{{ }^{2}}$ - wurde lange Zeit als eine wesentliche Ursache dieser fehlgeschlagenen Investitionen unterschätzt oder gar ignoriert. Erst seit etwa 15 Jahren kann empirisch belegt werden, dass Korruption in Verbindung mit extremer Armut zu einem Teufelskreis führt, der den Zugang zu zentralen Einrichtungen des öffentlichen Lebens wie Bildung und medizinischer Versorgung verwehrt. Diese Erkenntnis führte zu einem Umdenken in der entwicklungstheoretischen Diskussion, erforderte aber vor allem sofortige gezielte Handlungen in der entwicklungspolitischen Praxis. Wie kann Korruption bekämpft werden, damit entwicklungspolitische Ziele auch nachhaltig wirksam werden? In diesem Zusammenhang setzt die Weltbank auf Good Governance (GG), ein aktuelles entwicklungspolitisches Konzept, das sich an den liberal-demokratischen Kriterien Rechtssicherheit, Rechtsstaatlichkeit und Verantwortlichkeit orientiert. ${ }^{3}$ Dadurch wird Demokratieförderung zu einem zentralen Instrument, um nachhaltiges Wachstum zu erreichen. ${ }^{4}$ Doch inwiefern kann Demokratieförderung wirksam dazu beitragen, Korruption in EL einzuschränken?

* Nadja Memmer, International Fellow bei Socialdata (Brisbane), Institut für Transport- and Infrastukturforschung, Studien und Policies zu sozial-ökologischen Verhaltensänderungen; Studium der Politikwissenschaften, Volkswirtschaftslehre und Ethnologie in Heidelberg, Mainz und Granada mit Schwerpunkt Entwicklungspolitik; E-Mail: nadja_memmer@yahoo.de. Der Beitrag ist entstanden im Rahmen der Magisterarbeit an der Johannes Gutenberg-Universität Mainz. Ein herzlicher Dank geht vor allem an Herrn Dr. iur. LL.M. Philipp Dann, der mir diesen Beitrag ermöglicht hat. Weiterhin bedanke ich mich bei Frau Prof. Dr. Edeltraud Roller und Frau Prof. Dr. Ruth Zimmerling wie auch bei Herrn Dipl. Soz. Thies Böttcher.

1 United Nations Development Programme, Capacity Development: Empowering People and Institutions, Annual Report 2008, S. 13.

2 Joseph J. Senturia, Corruption, Political, in: Edwin R.A. Seligman (Hg.), Encyclopedia of the Social Sciences, New York 1931, S. 448 ff.

Franz Nuscheler, Lern- und Arbeitsbuch Entwicklungspolitik, Bonn 2005, S. 625.

Florian Grotz, Good Governance, in: Dieter Nohlen (Hg.), Lexikon Dritte Welt. Länder, Organisationen, Theorien, Begriffe, Personen, Reinbek, 12. Aufl. 2002, S. 341. 
Dazu werden in einer empirischen Querschnittsanalyse ${ }^{5} 149$ EL dahingehend untersucht, ob der Aufbau einer liberalen Demokratie - bestehend aus politischen Rechten und bürgerlichen Freiheiten - dazu führen kann, Korruption in EL zu reduzieren. In Anlehnung an die einschlägige Studie von Goldsmith (1999) wird diese Thematik anhand aktueller Daten neu aufgearbeitet. Der Artikel beginnt zunächst mit einem theoretischen Teil, der in die grundlegenden Zusammenhänge zwischen GG, liberaler Demokratie und Korruption einführt und damit die Basis der empirischen Analyse darstellt (A). Danach erfolgt die Beschreibung der methodischen Vorgehensweise zur Datenerhebung (B). Diese Daten werden sodann mittels statistischer Methoden analysiert und interpretiert (C). Abschließend soll auf Basis dieser Ergebnisse die Beantwortung der aufgeworfenen Fragestellung diskutiert werden (D). Ein Ausblick rundet den Beitrag ab (E).

\section{A. Good Governance, Demokratisierung und Korruption in Entwicklungsländern}

\section{Good Governance als entwicklungspolitische Strategie zur Korruptionsbekämpfung}

Good Governance (GG) ist eine entwicklungspolitische Strategie, die im Kontext zum Ende des Ost-West Konfliktes Anfang der 90er Jahre steht. Sie wurde zunächst von der Weltbank eingeführt und nachfolgend von den Industrieländern (IL) in modifizierter Form, d.h. nach deren jeweiligen staatlichen Interessen, übernommen. ${ }^{6}$ Im Kern meint GG die staatliche Gewährleistung von Rechtssicherheit, Rechtsstaatlichkeit und Verantwortlichkeit. ${ }^{7}$ Geht man nach Juan Linz davon aus, dass Rechtsstaat und Demokratie konform gehen, ${ }^{8}$ dann entspricht GG im politikwissenschaftlichen Verständnis gegenwärtigen Definitionen von liberalen Demokratien. ${ }^{9}$ Abbildung 1 veranschaulicht den Vergleich zwischen den Kriterien einer liberalen Demokratie und Good Governance.

Der Begriff „liberale Demokratie“ wird in diesem Beitrag als prozeduralistisch angesehen. Somit können auch Autokratien gewisse liberal-demokratischen Strukturen aufweisen, obwohl es sich laut Verfassungsnorm nicht um Demokratien handelt bzw. in dem jeweiligen Land de jure noch kein kompetitiver Wahlprozess vorhanden ist. ${ }^{10}$ Durch die Kopplung der Entwicklungshilfegelder an die Kriterien von GG zielt die internationale „Geber-

Eine Querschnittsanalyse untersucht eine bestimmte Anzahl von Ländern zu einem bestimmten Zeitpunkt.

Grotz, (Fn.4), S. 341f.

$7 \quad$ Nuscheler, (Fn. 3), S. 625.

8 Juan J. Linz und Alfred C. Stepan, Toward Consolidated Democracies, in: Journal of Democracy 7 (2) 1996, S. 14-33.

9 Larry Diamond, Developing Democracy Toward Consolidation, Baltimore and London 1999, S. $10 \mathrm{ff}$.

10

Diamond, (Fn. 9), S.10ff. 
Abbildung 1: Liberale Demokratie und Good Governance im Vergleich

\begin{tabular}{|l|l|}
\hline $\begin{array}{l}\text { Liberale Demokratie gemessen an Freedom } \\
\text { House Freedom in the World Indikatoren 2007 }\end{array}$ & $\begin{array}{l}\text { Good Governance gemessen an World Bank } \\
\text { Governance Indicators (WBGI) 2007 }^{12}\end{array}$ \\
\hline Electoral Process & $\begin{array}{l}\text { Government Effectiveness } \\
\text { Political Pluralism and Participation }\end{array}$ \\
Functioning of Government & Regulatory Quality \\
Associational and Organizational Rights & Political Stability and Absence of Violence \\
Personal Autonomy and Individual Rights & Voice and Accountability \\
Freedom of Expression and Belief & Rule of Law \\
Rule of Law & Control of Corruption \\
\hline
\end{tabular}

gemeinschaft “" ${ }^{13}$ darauf ab, die Partnerländer zu einer Verbesserung ihrer Regierungstätigkeit zu veranlassen und damit insbesondere der Korruption den Kampf anzusagen. ${ }^{14}$ „In a country with heavy corruption and poor governance, the priorities in anti-corruption efforts would then be to establish rule of law, strengthen institutions of participation and accountability, and limit government interventions to focus on core mandates." ${ }^{15}$ Ursache für die Einführung dieses Konzeptes war nach dem Ende des Ost-West-Gegensatzes die Obsoletheit der zuvor herrschenden entwicklungspolitischen Prämissen ${ }^{16}$ rein ideologischer und ökonomischer Natur. ${ }^{17}$ In diesem Zusammenhang musste sich die Weltbank eingestehen, dass ihre Strukturanpassungsprogramme aufgrund mangelnder Qualität der Regierungstätigkeit nicht die erwünschte Implementierung fanden. ${ }^{18}$ Stattdessen entwickelten sich vor allem in Afrika Gesellschaften mit einem schwachen Staat. Neben ,dem Mangel an qualifizierten Kadern, hinreichender verwaltungstechnischer Ausstattung und der Knappheit an Ressourcen“ ${ }^{19}$ ist Korruption mit ihren vielfältigen Ausprägungen ,eine wesentliche Ursa-

Freedom House, Freedom in the World Index Methodology 2007.

13

The World Bank, Governance Indicators 1996 - 2008, Washington D.C. 2009.

Nuscheler, (Fn. 3), S. 625.

14 Nuscheler, (Fn. 3), S. 625.

15 Anwar Shah and Jeff Huther, Anti-corruption Policies and Programs: A Framework for Evaluation, World Bank Policy Research Working Paper No. 2501 (2000).

16 Mehr dazu bei Ulrich Menzel, Das Ende der Dritten Welt und das Scheitern der großen Theorie, Frankfurt am Main 1997.

17

Grotz, (Fn. 4), S. 341.

18 Grotz, (Fn. 4), S. 341.

19 Nuscheler, (Fn. 3), S. 412. 
che für die geringe Handlungs- und Leistungsfähigkeit des „,schwachen Staates “20 „ „A wide consensus has also recently emerged that corruption is a symptom of failed governance and hence curtailing corruption requires addressing the causes of mis-governance." 21

Inwieweit korrupte Praktiken in einem Land vorherrschen, wird näherungsweise durch die Wahrnehmung international tätiger Geschäftsleute seit 1995 jährlich von der Nichtregierungsorganisation (NRO) Transparency International (TI) im Corruption Perceptions Index (CPI) erhoben. ${ }^{22}$ Der CPI zeigt auf einer Skala von 0 (Korruption wird als endemisch wahrgenommen) bis 10 (es wird nahezu keine Korruption wahrgenommen) an, dass alle erfassten Staaten ${ }^{23}$ bis zum gewissen Grad von Korruption betroffen sind. Allerdings weist der Index auch hohe bis endemische Korruption besonders in EL aus. ${ }^{24}$ Vor der Messbarkeit wahrgenommener Korruption gab es einen regen Diskurs über die Wirkungen, die von Korruption ausgehen. ${ }^{25}$ Erst Paolo Mauro konnte im Jahr 2005 zeigen, dass Korruption das ökonomische Entwicklungsniveau erheblich beeinflusst. ${ }^{26}$ Heute, nach über einem Jahrzehnt empirischer Korruptionsforschung kann man mit den Worten Franz Nuschelers sagen: "Korruption lähmt wie ein Krebsgeschwür, dessen Metastasen den ganzen politischen Körper befallen haben, das öffentliche Leben und ruiniert auch potenziell reiche Länder wie Zaire (Kongo), Nigeria, Kenia und Indonesien. Korruption machte einzelne Kleptokraten steinreich, aber ihre Völker bettelarm" ${ }^{27}$.

Deshalb und unter den gegebenen Umständen des Gebrauches von GG als Mittel zur (ökonomischen) Entwicklung (braucht Entwicklung Demokratie?) erscheint es von großem Interesse, empirisch $\mathrm{zu}$ untersuchen, wie weit liberal-demokratische Strukturen die Korruptionsbekämpfung beeinflussen. Dies führt dazu, die Zielfragestellung näher zu spezifizieren: Kann der Unterschied im Ausmaß der Korruption zwischen IL und EL neben dem ökonomischen Wachstum durch Demokratisierung begründet werden? Die nachfolgende theoretische Erörterung soll dazu weiteren Aufschluss geben und das Fundament der empirischen Analyse legen.

Nuscheler, (Fn. 3), S. 412.

Shah und Huther, (Fn. 15), S. 2.

Transparency International, Corruption Perception Index 2007, http://www.transparency.org/ policy_research/surveys_indices/cpi/2007.

Die nicht erfassten Staaten gehören laut TI vermutlich zu den korruptesten.

24 Nuscheler, (Fn. 3), S.412.

25 Bis zu diesem Zeitpunkt herrschte über Jahrzehnte ein reger Diskurs darüber, ob von Korruption nun positive (Funktionalisten) oder negative (Dysfunktionalisten) Wirkungen ausgehen. Vgl. Arthur A. Goldsmith, Slapping the grasping hand: Correlates of political corruption in emerging markets, in: American Journal of Sociology 58 (4) 1999, S. 866-883.

Paolo Mauro, Growth and Corruption, in: The Quaterly Journal of Economics (110) 1995, S. 681712. 


\section{Liberal-demokratische Institutionen als Kontrollmechanismen der Korruption}

Korruption „als Missbrauch öffentlicher Macht zu privatem Nutzen““28, bezeichnet eine Klasse von Normverletzungen. ${ }^{29}$ „Da es viele verschiedene normativ geregelte soziale Kontexte gibt, (...), gibt es demnach auch viele verschiedene denkbare Arten von Korruption“. ${ }^{30}$ Korruption stellt deshalb in der wissenschaftlichen Forschungsdiskussion aufgrund seiner Vielschichtigkeit ein fachübergreifendes Problem dar. Da in diesem Aufsatz der politische Aspekt von Korruption untersucht werden soll, bietet es sich für den theoretischen Rahmen an, Korruption anhand eines Prinzipal-Agent Modells zu untersuchen. „Incentive theory ${ }^{31}$ provides a conceptual framework for analysing the role played by public officials in designing positive incentives and defining penalties, and in shaping the institutional environment in which corruption takes place. “32

Das Prinzipal-Agent Modell gehört zu der Gruppe der rationalen Entscheidungstheorien, die unter dem Begriff der Neuen Politischen Ökonomie (NPÖ) zu finden sind. Die NPÖ zählt zu den wichtigsten theoretischen Strömungen der neueren Politikwissenschaft und unterstellt in Anlehnung an die moderne Volkswirtschaftslehre ein rationales Menschenbild des so genannten "homo oeconomicus". Er ist ein Nutzenmaximierendes und Kostenminimierendes Individuum, dessen Handlungsspielraum durch situative Restriktionen, wie institutionelle Regeln oder Rechtsnormen eingeschränkt wird. Soziale Beziehungen zwischen Individuen gelten dabei als sozialer Tausch, wobei der homo oeconomicus seine Entscheidungen unter anderem von den Entscheidungen anderer Individuen abhängig macht. $^{33}$

Korruption im Prinzipal-Agent Modell (PAM) wird im engeren Sinne definiert als ,an exchange of favours between two actors, an agent and a client“"34. Nach Zimmling stellt Korruption in diesem Kontext eine Tauschbeziehung mit zwei besonderen Merkmalen dar: „(1) eine Seite des Tauschs ist eine Amtshandlung, und (2) der Amtsträger ist zur Herstellung dieser Tauschbeziehung nach den zugrunde liegenden Regeln des betreffenden normativen Systems nicht autorisisiert. “ ${ }^{35}$ Das PAM unterliegt der Prämisse, dass öffentliche

28

29

30

31

32

33

34

35

Senturia, (Fn. 2), S. 448ff.

Ruth Zimmerling, Politische Korruption: begrifflich-theoretische Einordnung, in: Ulrich von Alemann (Hg.), Dimensionen politischer Korruption, Beiträge zum Stand der internationalen Forschung, Wiesbaden 2005, S. 77.

Zimmerling, (Fn. 29), S. 77.

Mit ,incentive theory“ ist die rationale Entscheidungstheorie gemeint.

Jens C. Andvig/ Odd-Helge Fjeldstad / Inge Amundsen / Tone Sissener / Tina Søreide, Corruption, A Review of Contemporary Research, NUPI Report 268 2001, Mimeo, S. 79.

Ulrich Druwe, Politische Theorie, Neuried 1995, S. 306.

Andvig et al., (Fn. 32), S. 79.

Zimmerling, (Fn. 29), S. 79. Durch die zweite Definitionsbedingung wird Korruption relativ zum jeweils geltenden normativen System assoziiert. Dadurch wird das Problem des Ethnozentrismus in der Definition von Korruption entschärft (vgl. Ernesto Garzón Valdes, Korruption - Zur syste- 
Organisationen in Hierarchien organisiert sind, und demzufolge der Beziehung zwischen untergeordneten Angestellten und deren Vorgesetzten bei der Gewährleistung eines effizienten Organisationsprozesses eine entscheidende Rolle zugeschrieben wird. Wird diese Beziehung von einem der beiden Beteiligten missbraucht, indem er sein Amt zur persönlichen Bereicherung benutzt, so wird ein effektiver Arbeitsablauf gestört und es kann vor allem für Dritte, etwa Steuerzahler, zu erheblichen Schäden kommen. ${ }^{36}$ Alemann zählt zu dieser Austauschlogik die folgenden sieben Komponenten: ${ }^{37}$

„1. Der Nachfrager (der Korrumpierende) will 2. ein knappes Gut (Auftrag, Konzession, Lizenz, Position), 3. das der Anbieter, der Entscheidungsträger in einer Organisation oder Behörde, also der Korrumpierte, vergeben kann. 4. Er erhält einen persönlichen verdeckten Zusatzanreiz (Geld oder geldwerte Leistung) für die Vergabe über den normalen Preis hinaus und 5. verstößt damit gegen öffentlich akzeptierte Normen und 6. schadet damit Dritten, Konkurrenten und/oder dem Gemeinwohl. 7. Deshalb findet Korruption versteckt, im Verborgenen statt.“

Die renommierte Korruptionsforscherin Rose-Ackerman ${ }^{38}$ projiziert dieses Verhaltensmuster auf die politische Ebene und folgert: „The relationship links at least two actors. On the one hand, the superior expresses a set of preferences which specify desired outcomes. On the other hand, there is an agent, whom the superior has directed to achieve these outcomes. Thus democratic legislators are the agents of voters; agency heads, of legislators; and bureaucrats, of agency heads." 39 Anhand dieses rationalen Verhaltensmodells leitet sie freie, faire und konkurrierende Wahlen, Gewaltenteilung, unabhängiges Rechtssystem und bürgerliche Freiheiten als zentrale politische Institutionen, die korruptes Verhalten im politischen Prozess einschränken können, ab. ${ }^{40}$ Auch wenn Kritiker bei einer solchen Anwendung des PAM vor einer Überstrapazierung warnen und eine Differenzierung zwischen Korruption in privaten und öffentlichen Organisationen postulieren, ${ }^{41}$ so gelten die nachfolgend skizzierten Ausführungen in der internationalen Korruptionsforschung als

mischen Relativität eines universalen Phänomens, in: Harald Bluhm / Karsten Fischer (Hrsg.), Sichtbarkeit und Unsichtbarkeit der Macht. Theorien politischer Korruption. Baden-Baden 2000, 115-138; Kurt Weyland, The Politics of Corruption in Latin America, in: Journal of Democracy 9 (2) 1998, S. 108-121; Robin Theobald, Corruption, Development and Underdevelopment. Durham, N.C. 1990.)

Ulrich von Alemann, Politische Korruption: Ein Wegweiser zum Stand der Forschung, in: Ulrich von Alemann (Hg.), Dimensionen politischer Korruption, Beiträge zum Stand der internationalen Forschung, Wiesbaden 2005, S. 29.

Von Alemann, (Fn. 36), S. 31.

38 Susan Rose-Ackerman, Corruption and Government: Causes, Consequences and Reform, Cambridge 1999, S. 127-144. 
bahnbrechend ${ }^{42}$ und werden deshalb in diesem Aufsatz als theoretisches Fundament verwendet.

Rose-Ackerman relativiert in ihren Ausführungen zunächst den Einfluss von reinen Wahldemokratien auf die Einschränkung korrupten Verhaltens. Zwar sorge der politische Wettbewerb zwischen mindestens zwei sich konkurrierenden Parteien dafür, etwaiges Fehlverhalten des jeweiligen Gegners aufzudecken. Werden Freiheit, Fairness und Konkurrenz im Wahlprozess jedoch nicht eindeutig gewährleistet, wie dies häufig in ,defekten Demokratien“ der Fall ist, besteht die Gefahr, dass Wahldemokratien zusätzliche Korruption begünstigen können. Dementsprechend wird gefolgert, dass die Einführung von Wahldemokratien als nicht ausreichend erscheint, um Korruption institutionell zu kontrollieren. Vielmehr bedürfe es drei weiterer zentraler politischer Institutionen, wie die der Gewaltenteilung, eines unabhängigen Rechtssystems und der bürgerlichen Freiheiten, deren Funktionen zur Korruptionskontrolle nachfolgend kurz skizziert werden.

Die Trennung zwischen Exekutive und Legislative (Horizontale Gewaltenteilung) stellt durch die Aufhebung einer absoluten Machtspitze einen Kontrollprozess zwischen beiden Institutionen dar, der korruptes Verhalten einschränken kann. Ein politisch unabhängiges Rechtssystem kann ferner Korruption erschweren, Reformen voranbringen und rechtliche Normen sichern. Opfer korrupten Verhaltens ermöglicht dies einerseits, sich vor Gericht Gehör verschaffen. Anderseits erhöht sich das Risiko der strafrechtlichen Verfolgung korrupter Vergehen signifikant, wenn das Gerichtssystem selbst unabhängig und kompetent ist. Ist ein Staat weiterhin in der Lage, bürgerliche Freiheitsrechte wie Informations-, Presse-, Meinungs- und Versammlungsfreiheit zu gewährleisten, dann kann öffentlicher Druck zu einem Ventil werden, das korruptes Verhalten entweder durch individuelle oder kollektive Beschwerden aufdecken kann. ${ }^{43}$

\section{Generierung der Hypothesen}

Wie gezeigt, kann Korruption durch die politischen Institutionen freie, faire und konkurrierende Wahlen, Gewaltenteilung, unabhängiges Rechtssystem und bürgerliche Freiheiten eingeschränkt werden. Verglichen mit einer Demokratiedefinition nach Diamond (1999) stellen diese Faktoren auch die zentralen Elemente der liberalen Demokratie dar: ${ }^{44}$

„Beyond parties and elections, citizens have multiple, ongoing channels for expression and representation of their interests and values, including diverse, independent associations and movements, which they have the freedom to form and join. There are alternative sources of information (including independent media) to which citizens have (politically) unfettered access. Individuals also have substantial freedom of belief, opinion, discussion, speech, publication, assembly, demonstration, and petition. Individual and group liberties are effectively protected by an inde- 
pendent, nondiscriminatory judiciary, whose decisions are enforced and respected by other centers of power".

Im Folgenden wird deshalb nicht weiter von politischen Institutionen, sondern von liberaldemokratischen Strukturen gesprochen, welche die Korruption eines Landes beeinflussen können. Aufgrund dieser Annahmen werden folgende Hypothesen abgeleitet.

H1: Je liberal-demokratischer ein Land, im Sinne der liberalen Demokratiedefinition nach Diamond (1999), desto niedriger die Korruption.

H2: Da die Elemente einer liberalen Demokratiedefinition nach Diamond (1999) ein wesentlicher Bestandteil des entwicklungspolitischen Konzeptes von GG sind, erweist es sich als effiziente Strategie zur Korruptionsbekämpfung.

Nachfolgend kann nun überprüft werden, ob diese Hypothesen auch für die ausgewählte Gruppe der EL zutreffend ist. Dazu werden im nächsten Abschnitt zunächst die Methoden der Datenerhebung beschrieben.

\section{B. Die Datenerhebung}

Bisher wurden neben der Frage, inwiefern Demokratieförderung dazu beiträgt, Korruption in EL zu reduzieren, auch die theoretischen Zusammenhänge zwischen Korruption, Demokratie und GOOD GOVERNANCE in EL erläutert. Mit einer Zunahme an liberal-demokratischen Strukturen konnte auch ein niedrigeres Korruptionsniveau beobachtet, und demnach das entwicklungspolitische Konzept von GG als effiziente Maßnahme der Korruptionsbekämpfung betrachtet werden. Die empirische Überprüfung dieser Hypothesen für die ausgewählte Gruppe der EL, erfordert zunächst eine gültige (valide) und zuverlässige (reliabel) Datenerhebung für die Messgrößen Demokratie und Korruption eines jeden EL. Da es sich dabei um komplexe und aufwändige Messvorgänge handelt, die im Rahmen der hier vorliegenden Arbeit nicht in Eigenleistung ausgeführt werden können, wird auf ausgesuchte Datenerhebungen internationaler Nichtregierungsorganisationen (NROs) zurückgegriffen.

\section{Datenerhebung in Entwicklungsländern}

Die Ländergruppe der EL als solche in einem einzigen Begriff zusammen zu fassen, bringt enorme Schwierigkeiten und erhebliche berechtigte Kritik mit sich. ${ }^{45}$ Allerdings kann dies in der vorliegenden Analyse nicht zum Diskussionspunkt werden. Vielmehr soll auf den

Sowohl die Terminologie als auch die Kategorisierung von EL sind nicht unumstritten. Dem Begriff „Entwicklungsland“ liegt kein theoretisches Konzept zugrunde und definiert sich vor allem darüber, ob Entwicklung als Stadium oder als strukturelle Integration in die internationale Arbeitsteilung angesehen wird (vgl. Dieter Nohlen, Entwicklungsländer, in: Dieter Nohlen (Hg.), Lexikon Dritte Welt. Länder, Organisationen, Theorien, Begriffe, Personen, Reinbek bei Hamburg 2002, S. 233-235). Trotz berechtigter Kritik im Vergleich zu früheren Bezeichnungen wie „Dritte Welt" erscheint er allerdings als ,unbedenklicher Sammelbegriff“ und ,,besitzt allgemeine Gültigkeit", vgl. Nuscheler, (Fn.3), S. 99. 
kritischen Diskurs verzichtet werden, da es für die diesen Beitrag lediglich interessant ist, ob ein Land in entwicklungspolitischen Dimensionen ein Geber- oder Empfängerland von Entwicklungshilfegeldern ist. Dementsprechend werden nachfolgend alle Länder, die Empfänger von Entwicklungshilfegeldern sind, als EL bezeichnet. ${ }^{46}$ Um EL als solche zu identifizieren, wird gewöhnlich die Klassifizierung der Weltbank verwendet, die jährlich das Pro-Kopf-Einkommen nahezu aller Länder der Welt erhebt ${ }^{47}$ und dieses in die Kategorien niedriges, niedriges mittleres, höheres mittleres und hohes Pro-Kopf-Einkommen einteilt. ${ }^{48}$ Dabei werden alle Länder mit nicht hohem Einkommen von der Weltbank als EL bezeichnet. Durch diese einheitliche, wenn auch aus entwicklungstheoretischer/-soziologischer Sicht sehr eindimensionale Abgrenzung, ${ }^{49}$ kann gewährleistet werden, dass alle Länder, die potentiell mit den Vergabekriterien von GG in Berührung kommen, in die Analyse aufgenommen werden. Die Weltbank klassifizierte 2007 demzufolge 149 EL, d.h. 53 Länder mit niedrigem (weniger als US \$905), 55 Länder mit niedrigem mittleren Einkommen (US \$906 - \$3.595) und 41 mit höherem mittlerem (US \$3.596 - \$11.115) ProKopf-Einkommen. ${ }^{50}$

\section{Datenerhebung zur Messung von Korruption in Entwicklungsländern}

Um Korruption, definiert als „,der Missbrauch öffentlicher Macht zu privatem Nutzen“ ${ }^{51}$, in diesen 149 EL zu untersuchen, bieten die Messdaten des renommierten Corruption Perception Index $(\mathrm{CPI})^{52}$ die beste Validität, um das theoretische Konstrukt der Korruption so exakt wie möglich abzubilden. Der hier verwendete CPI 2007 misst das Ausmaß der Korruptionswahrnehmung in 146 der 149 EL. ${ }^{53}$ Er ist eine Zusammenfassung aus repräsentativen Umfragen (14 Erhebungen von 12 unabhängigen Institutionen) an Geschäftsleuten und Schätzungen von Länderanalysten, die in den Jahren 2006 und 2007 erstellt oder veröffentlicht wurden. Durch die Verwendung von Quellen, die auf unterschiedlichen Stichpro-

46

47

48

49

Die Kritik an der Eindimensionalität der Ländereinteilung der Weltbank kann als eigenständige Abhandlung betrachtet werden. Ein alternatives Messinstrument, das auch politische und soziokulturelle Dimensionen abbildet ist der Human Development Index der Vereinten Nationen, vgl. dazu Nuscheler, (Fn. 5), S. 190 f.

50

51

52

53

In der internationalen Entwicklungszusammenarbeit (EZ) wird in diesem Zusammenhang von Partnerländern gesprochen.

Diese Daten werden anhand der Welt Bank Atlas Methode berechnet (Vgl. The World Bank, Atlas Method, Washington D.C. 2007.

Nuscheler, (Fn. 3), S. 103.

The World Bank, Country Classification, Washington D.C. July 2007, download vom 31.08.2008.

Senturia, (Fn. 2), S. 448ff.

Transparency International, (Fn. 22).

Johann Lambsdorff, The Methodology of the Corruption Perceptions Index 2007, complete version, http://www.transparency.org/policy_research/surveys_indices/cpi/2007/methodology, S. 1. 
benplanungen und Methodologien basieren, soll das Verständnis des tatsächlichen Korruptionsniveaus erhöht werden. ${ }^{54}$ Die Datenauswahl richtet sich nach strikten Vorgaben, nach denen entschieden wird, ob sich eine Quelle zur Aufnahme qualifiziert und letztendlich verwendet werden soll. Demnach muss bei diesen Quellen zum einen ein Länderranking vorhanden sein, und zum anderen das komplette Ausmaß von Korruption gemessen werden können. ${ }^{55}$ Eine solche subjektive d.h. auf Wahrnehmung basierende Datenerhebung von Korruption auf Basis des CPI ist nicht unumstritten, ${ }^{56}$ gilt aber in der empirischen Korruptionsforschung als das zuverlässigste Instrument. ${ }^{57}$ Eine viel versprechende Alternative zur Problematik der Gewährleistung einer zuverlässigen (reliablen) Datenerhebung zur Korruption stellt das Globale Korruptionsbarometer (GCB) von TI dar, dessen Datenumfang bislang keine große Querschnittsanalyse ermöglicht. Das Barometer evaluiert anhand einer repräsentativen Meinungsumfrage, wie und wo Bürger dem Einfluss von Korruption tatsächlich begegnen. ${ }^{58}$ Wichtig für die Reliabilität der Daten des CPI (zwei voneinander unabhängige Messinstrumente führen zum gleichen Ergebnis) ist deshalb, dass es nach den Ergebnissen aus dem Jahr 2007 eine starke Korrelation zwischen den Erfahrungen der Bürger mit Korruption (GCB) und der wahrgenommenen Korruption (CPI) gibt. In denjenigen Ländern, in denen Geschäftsleute, Länderanalysten und Experten Korruption als weit verbreitet wahrnehmen, bezahlt ein höherer Prozentsatz der Bevölkerung Bestechungsgelder für eine Dienstleistung. Dies deutet an, dass die Expertenmeinung mit den Erfahrungen der Bürger mit Korruption im öffentlichen Sektor verbunden ist. ${ }^{59}$

Die wahrgenommene Korruption eines Landes, ermittelt mit dem CPI 2007, wird in dieser Analyse mit der Variablen ,wahrgenommene Korruption“ beschrieben und analysiert. Die Variable erstreckt sich auf einer Skala von Null (Korruption wird als endemisch wahrgenommen) bis 10 (es wird eigentlich keine Korruption wahrgenommenen) und erfasst 146 der 149 klassifizierten EL ${ }^{60}$. Der kleinste Wert der Verteilung liegt bei 1,4 (Myanmar und Somalia) und der größte bei 7 (Chile), was insgesamt als eine sehr hohe wahrgenommene Korruption in EL interpretiert werden kann.

54

55

56

Lambsdorff, (Fn. 53), S. 1.

Lambsdorff, (Fn. 53), S. 2.

Subjektive Erhebungen via Fragebögen (wahrgenommene Korruption) können die Wirklichkeit nicht akkurat abbilden, bieten aber einen zuverlässigen Näherungswert. Objektive Datenerhebung (harte Daten) hingegen erfassen nicht Korruption per se, sondern die Effektivität und Kapazität des Justizsystems eines Landes durch die jeweiligen Korruptionsanklagen. Damit wird die Messung ungültig, vgl. Johann Lambsdorff, The Institutional Economics of Corruption and Reform: Theory, Evidence, and Policy, Cambridge 2005.

Björn Frank, Zehn Jahre empirische Korruptionsforschung, in: Vierteljahreshefte zur Wirtschaftsforschung 73 (2) 2004, S.184-199.

Transparency International, Report on the Global Corruption Barometer 2007.

Transparency International, (Fn. 58), S.10.

Von den 149 EL werden Mayotte, Nördliche Mariannen Inseln, Palau nicht vom CPI 2007 erfasst. 
Länder mit niedrigem Einkommen haben die höchste durchschnittlich wahrgenommene Korruption (2,7). Daran schließen sich Länder mit niedrigem mittlerem Einkommen $(3,2)$ an. In Ländern mit höherem mittlerem Einkommen hingegen wird die Korruption durchschnittlich am wenigsten wahrgenommen $(4,1) .{ }^{61}$ Daraus kann gefolgert werden, dass sich die Länder hinsichtlich ihrer wahrgenommenen Korruption in den jeweiligen Einkommensklassen unterscheiden. Diese Beobachtungen weisen damit auf den bereits mehrfach bestätigten Zusammenhang zwischen ökonomischen Entwicklungsniveau und Korruption hin. ${ }^{62}$

\section{Datenerhebung zur Messung von Demokratie in Entwicklungsländern}

Das Konzept von GG enthält überwiegend Elemente liberal-demokratischer Strukturen, die wiederum aus politischen Rechten und zivilen Freiheiten bestehen. Liberal-demokratische Strukturen können am besten durch die Datenerhebung des „Freedom in the World Index“ (FWI) der NRO Freedom House (FH) gemessen werden. Der FWI ist eine jährliche repräsentative Meinungsumfrage zum Stand der Verfassungsrealität von politischen Rechten und bürgerlichen Freiheiten. Dabei leiten sich die Fragen aus den relevanten Passagen der universellen Menschenrechtserklärung ab. Eine Orientierung an diesen Standards hat den Vorteil, dass sie auf alle Länder und Territorien angewendet werden kann, ungeachtet ihrer geographischen Lage, ethnischen oder religiösen Zusammensetzung oder des ökonomischen Entwicklungsniveaus. ${ }^{63}$ Der Index misst Freiheit (die Möglichkeit, spontan auf viele verschiedene Arten außerhalb der Kontrolle der Regierung und anderer Machtzentren zu agieren) entsprechend den beiden weiten Kategorien: ,political rights“ und ,civil liberties“. Die beiden daraus resultierenden Teil-Indizes ,political rights“ und „civil liberties“ setzen sich jeweils aus drei (Wahlprozess; Politische Partizipation; Leistungsfähigkeit der Regierung) bzw. vier Subkategorien (Meinungs- und Glaubensfreiheit; Versammlungs- und Organisationsrechte; Rechtssystem; persönliche Autonomie und individuelle Rechte) zusammen. ${ }^{64}$ Aus diesen Maßzahlen werden die evaluierten Länder anschließend von FH in die drei Kategorien frei, teilweise frei und nicht frei unterteilt. ${ }^{65}$ Auf dieser Basis ermöglicht FH ferner die Identifizierung von Ländern mit Wahldemokratien, d.h. Länder, die

61

Eigene Berechnungen nach Transparency International, (Fn. 22) und The World Bank, (Fn. 50).

Rafael La Porta / Florencio Lopez de Silanes / Andrej Shleifer / Robert Vishny, The quality of government, in: Journal of Economics, Law and Organisation 15 (1) 1999, S. 222-279; Alberto Ades und Rafael di Tella, Rents, competition, and corruption, in: American Economic Review 89 (4) 1999, S. 982-93; Goldsmith, (Fn. 27); Daniel Treisman, The Causes of Corruption: A CrossNational Study, in: Journal of Public Economics (76) 2000, S. 399-457; Daniel Treisman, What have we learned about the causes of corruption from ten years of cross-national research?, 2006, http://www.sscnet.ucla.edu/polisci/faculty/treisman/what_have_we_learnHg.pdf.

Freedom House, (Fn. 10).

Freedom House, Freedom in the World, Country Subscores 2007.

Freedom House, Freedom in the World Combined Average Ratings-Independent Countries 2007. 
mindestens einen kompetitiven Wahlprozess zwischen zwei Parteien haben. ${ }^{66}$ Der FWI erscheint folglich für diese Analyse als bestes Messinstrument, da er die Dimensionen einer liberalen Demokratiedefinition nach Diamond nahezu identisch abbildet und damit das Validitätskriterium erfüllt.

Ein Vergleich mit dem alternativen Messinstrument „Demokratie-Status Index“ des Bertelsmann Transformationsindex (BTI) ${ }^{67}$ zeigt eine hohe Korrelation mit dem FWI (Reliabilität). ${ }^{68}$ Der Demokratie-Status Index des BTI ist ein seit 2003 sich etablierender Ansatz, der sich speziell mit der Messung von liberal-demokratischen Strukturen in EL auseinandersetzt. Er besteht aus fünf Kriterien zum Status der rechtsstaatlichen Demokratie (bewertet anhand von 18 Einzelfragen). Ihm liegt ein Demokratieverständnis zugrunde, das über die Durchführung von freien Wahlen hinausgeht und das tatsächliche Funktionieren des demokratischen Systems in den Mittelpunkt rückt. Dies drückt sich in den Kriterien zu Rechtsstaatlichkeit, institutioneller Stabilität und in den Bewertungen zur Stärke von Zivilgesellschaft und politischer Partizipation aus. ${ }^{69}$ Inhaltlich erreicht der Demokratie-Status Index allerdings nicht den Umfang des FWI, weshalb er in der folgenden Analyse als Messinstrument gegenüber dem FWI ausscheidet. Ein weiterer wesentlicher Vorteil des FWI besteht darin, dass er sich auf die Verfassungswirklichkeit der erfassten Staaten bezieht, und damit nicht nur die Institutionalisierung von Rechten, sondern deren tatsächliche Gewährleistung analysiert. Dies hat zwar den Nachteil, dass die Ländereinschätzungen aufgrund von Experteneinschätzung und nicht durch einen objektiven Vorgang erhoben werden. Dieser kann jedoch aufgrund höherer Information in Kauf genommen werden. Problematisch ist ferner, dass sich eine Demokratiemessung nach FH eng an der US amerikanischen Demokratie orientiert. Da dies aber ein generelles Problem der entwicklungspolitischen Diskussion ist, und die politischen Handlungsempfehlungen der Demokratieförderung eben genau durch diese Daten legitimiert werden, steht diese Tatsache nicht in Zusammenhang mit der Qualität dieser Analyse. Ein weiteres Problem, welches die Aussagekraft der Ergebnisse allerdings erheblich erschweren wird, besteht darin, dass FH bei der Bewertung der als frei kategorisierten Länder die Abwesenheit von Korruption selbst voraussetzt (Endogenitätsproblem). Dies kann zu Verzerrungen in den Ergebnissen der Analyse führen. Aufgrund dieser hochgradigen Endogenität liberal-demokratischer Strukturen ist dieses Problem in der nachfolgenden Analyse nicht wegzudenken und muss deshalb bei der Interpretation der Daten Berücksichtigung finden.

66

67

68

69

Freedom House, Freedom in the World Electoral Democracies 2007.

Bertelsmann Stiftung, Bertelsmann Transformationsindex 2006: Demokratie-Status Index, http://www.BTI_2006_Broschuere_D_gesamt.pdf.

Eigene Berechnungen nach Freedom House, Freedom in the World, Country Subscores 2006; Bertelsmann Stiftung, (Fn. 67).

Bertelsmann Stiftung, (Fn. 67). 
Anhand der Daten des FWI 2007 können die liberal-demokratischen Strukturen von 144 der 149 von der Weltbank als EL gemessen werden. ${ }^{70}$ Aus diesen Daten wird aus dem Mittelwert der beiden Teilindikatoren ,political rights“ und „,civil liberties“ die unabhängige Variable ,liberal-demokratische Strukturen“ gebildet. ${ }^{71}$ Die daraus resultierende Skala von 1 bis 7 wurde anschließend umgedreht, um die Verwendung des positiven Demokratiebegriffs besser zu veranschaulichen. Demzufolge entspricht ein Wert von 1 einem Land, das weder politische Rechte noch zivile Freiheiten aufweist. In Ländern mit einem Wert von 7 sind diese beiden Komponenten nahezu vollständig und effizient ausgebildet. Anhand dieser Daten kann beobachtet werden, dass 33 Prozent der messbaren 144 EL als frei bezeichnet werden können. Von den teilweise freien EL werden 23 Prozent zumindest als Wahldemokratie klassifiziert. 17 Prozent hingegen entsprechen nicht den Kriterien einer Wahldemokratie. Dafür weisen sie aber einen gewissen Grad an anderen liberal-demokratischen Strukturen auf. 27 Prozent aller EL zählen zur Kategorie nicht frei. ${ }^{72}$ Aufgeteilt nach Einkommensklassen weisen EL mit niedrigem Einkommen durchschnittlich einen Wert der Variable liberal-demokratische Strukturen von 3,6 auf. Länder mit niedrigen mittleren Einkommen hingegen haben durchschnittlich eine wahrgenommene Korruption von 4,1, Länder mit mittlerem hohem Einkommen von 4,5. Ein Mittelwertvergleich dieser Stichproben ergibt, dass ein höheres ökonomisches Entwicklungsniveau mit einer Zunahme liberaldemokratischer Strukturen korrespondiert. ${ }^{73}$ Allerdings konnte ein eindeutiger Zusammenhang bislang empirisch nicht bestätigt werden. ${ }^{74}$

\section{Datenanalyse}

Im vorigen Kapitel wurden die Indikatoren TI-CPI (wahrgenommene Korruption) und FHFWI (liberal-demokratische Strukturen) vorgestellt, sowie deren Gültigkeit (Validität) und Zuverlässigkeit (Reliabilität) für die Messung in den 149 EL diskutiert. Anhand dieser Daten kann nun die aufgestellte Hypothese, dass in EL mit einer Zunahme an liberal-demokratischen Strukturen ein niedrigeres Korruptionsniveau verbunden ist, und demnach das entwicklungspolitische Konzept von GG als effiziente Maßnahme der Korruptionsbekämpfung betrachtet werden kann, mittels statistischer Analysen überprüft werden.

Die Demokratiemessung von Freedom House 2007 (Fn. 66 und 67) umfasst nicht Amerikanisch Samoa, Mayotte, Myanmar, Nördliche Mariannen Inseln sowie West Bank und Gaza.

Die kombinierten Werte für jedes Land finden sich unter: Freedom House, (Fn. 65). 


\section{Deskriptive Analyse}

Die Beziehung zwischen liberal-demokratischen Strukturen und Korruption konnte in der empirischen Korruptionsforschung bislang noch nicht eindeutig geklärt werden. ${ }^{75}$ Im Mittelpunkt der zahlreichen Analysen steht vor allem der politische Wettbewerb, ${ }^{76}$ wie er der Schumpeter'schen Demokratiedefinition zugrunde liegt. Problematisch ist hierbei einerseits, dass die Definition, die sich lediglich an einem Mindestmaß demokratischer Institutionen orientiert, die Relevanz bürgerlicher Freiheitsrechte ausklammert. ${ }^{77}$ Andererseits beurteilen Kritiker die Eignung der jeweils verwendeten Indikatoren zur Messung des politischen Wettbewerbs als teilweise fragwürdig. ${ }^{78}$ Eigens für EL wurde der Zusammenhang zwischen liberal-demokratischen Strukturen und Korruption im Jahr 1999 von Goldsmith untersucht. ${ }^{79}$ Er analysierte ökonomische Liberalisierung, Demokratisierung und administrative Zentralisierung mit politischer Korruption in einer Querschnittstudie von 34 Ländern mit niedrigen und mittleren Einkommen. Dabei identifizierte er einen zwar statistisch signifikanten, allerdings schwachen Zusammenhang zwischen wahrgenommener Korruption (gemessen durch den TI-CPI von 1996) und Demokratisierung (gemessen mit dem FH-FWI Teilindikator "political rights" von 1996/1997), den er nach Ausschluss des ökonomischen Entwicklungsniveaus als Kontrollvariable nicht als statistisch signifikant bestätigt werden konnte. $^{80}$

Eine im Jahr 2007 wesentlich breitere Datenbasis von 143 EL gibt deshalb genug Anlass, den Zusammenhang zwischen liberal-demokratischen Strukturen und Korruption erneut aufzurollen. Dabei soll ferner die Messung liberal-demokratischer Strukturen anhand der Synthese der beiden FH-FWI Teilindikatoren ,political rights“ und „,ivil liberties“ des FWI vorgenommen werden, die bereits unter D.III näher erläutert wurden. Um mit der Überprüfung zu beginnen, werden in einem ersten Schritt die Daten anhand deskriptiver Analysen untersucht. Dazu kann gezeigt werden, dass die 47 freien EL mit einer durchschnittlich wahrgenommenen Korruption von 4,1 eine geringer wahrgenommene Korrup-

Philip Manow, Politische Korruption und politischer Wettbewerb: Probleme der quantitativen Analyse, in: Ulrich von Alemann (Hg.), Dimensionen politischer Korruption, Beiträge zum Stand der internationalen Forschung, Wiesbaden 2005, S. 251-266.

Gabriella R. Montinola und Robert W. Jackman, Sources of Corruption: A Cross-Country Study, in: British Journal of Political Science 32 (2002), S. 147-170; Treisman, (Fn. 62); Ugo Panizza, Electoral Rules and Corruption, in: Transparency International: Global Corruption Report 2003, S. 317-319; Torsten Persson, Guido Tabellini und Francesco Trebbi, Electral Rules and Corruption, in: Journal of the European Economic Association 1, 4 (2003), S. 958-989.

77

78

79

80

Diamond, (Fn. 9), S. 10ff.

Manow, (Fn. 75), S. 251-266.

Goldsmith, (Fn. 25).

Goldsmith, (Fn. 25). 
tion verzeichnen als die 57 nur teilweise freien $(2,8)$ und die 39 nicht freien $(2,5) .{ }^{81}$ Einen präziseren Eindruck dieser Beobachtung erhält man mit Hilfe eines Streudiagramms ${ }^{82}$. Es zeigt, dass niedriger wahrgenommene Korruption mit einer Verbesserung an liberal-demokratischen Strukturen in Relation steht. ${ }^{83}$ Damit wird die Existenz eines möglichen Zusammenhangs zwischen liberal-demokratischer Struktur und wahrgenommener Korruption angedeutet. Betrachtet man sich jedoch die größte Gruppe, nämlich die teilweise freien EL und unterscheidet zwischen Wahldemokratie und Nicht-Wahldemokratie, so zeigt sich, dass teilweise freie EL ohne Wahlprozess eine durchschnittlich geringere wahrgenommene Korruption $(2,9)$ aufweisen als teilweise freie EL mit Wahlprozess $(2,7) .{ }^{84}$ Diese ersten nicht-linearen Beobachtungen sollen nun anhand der nachfolgenden Analyse näher untersucht werden.

\section{Lineare Einfachregression}

Diese ersten deskriptiven Befunde werden nun in einem zweiten Schritt mittels linearer Einfachregression, analysiert. ${ }^{85} \mathrm{Sie}$ ist das gängige Verfahren zur Untersuchung eines statistischen Zusammenhangs zwischen einer abhängigen Variablen (x) und einer unabhängigen Variablen (y). Die für diese Studie durchgeführten linearen Regressionstechniken bestehen aus komplexen rechnerischen (formalen) und graphischen Auswertungsverfahren, die in diesem Rahmen nicht ausführlich beschrieben werden können. ${ }^{86}$ Deshalb werden im Folgenden lediglich die zentralen Ergebnisse der durchgeführten Analysen herausgearbeitet.

Die erste Untersuchung des einfachen linearen Regressionsmodells, das die Beziehung zwischen wahrgenommener Korruption (x) und liberal-demokratischen Strukturen (y) zeigt, dass der gesamte Verlauf der wahrgenommenen Korruption in Abhängigkeit der Ausprägung liberal-demokratischer Strukturen, eine näherungsweise immer weiter zunehmende

81

Eigene Berechnungen nach The World Bank, (Fn. 50); Transparency International, (Fn. 22); Freedom House, (Fn. 67).

In einem Streudiagramm wird jede Beobachtung (hier jedes EL) in einem zweidimensionalen Raum abgebildet, wobei die Koordinaten jedes Punktes aus den Werten einer Beobachtung auf den entsprechenden Variablen resultieren (Vgl. Ulrich Kohler / Frauke Kreuter, Datenanlyse mit Stata. Allgemeine Konzepte der Datenanalyse und ihre praktische Anwendung, München/Wien 2008, S. 185).

Eigene Berechnungen nach The World Bank, (Fn. 50); Transparency International, (Fn. 22); Freedom House, (Fn. 65).

Eigene Berechnungen nach The World Bank, (Fn. 50); Transparency International, (Fn. 22); Freedom House, (Fn. 66 und 67).

Eine gute Einführung in die Regressionstechnik bietet Klaus Backhaus / Bernd Erichson / Wulff Plinke / Rolf Weiber, Multivariante Analysemethoden. Eine anwendungsorientierte Einführung, Berlin u.a. 2003, S. 46-77.

Ausführliche Darstellung aller durchgeführten Analysen in der Magisterarbeit der Verf'in., als PDF verfügbar. 
Reduzierung der wahrgenommenen Korruption impliziert. Die Analyse weist allerdings auch daraufhin, dass diese Zunahme enorme Schwankungen aufweist, deren Ursachen nicht geklärt werden können. Das Modell wird damit als nicht-linear charakterisiert und unterliegt deshalb einer schweren Annahmeverletzung. Sie muss korrigiert werden, indem die Variable liberal-demokratische Strukturen quadriert wird.

Da die Verteilung der abhängigen Variablen wahrgenommene Korruption im Modell nicht symmetrisch ist, besteht ferner die Gefahr einer ineffizienten Schätzung. Deshalb wird das Modell einer so genannten robusten Schätzung unterzogen.

Das aus diesen Modifizierungen resultierende als robust geschätzte Modell mit 143 EL zeigt einen statistisch signifikanten Zusammenhang zwischen den beiden untersuchten Variablen liberal-demokratische Strukturen und wahrgenommene Korruption. Weiterhin zeigt dieses Modell, dass rund 40 Prozent der Streuung (Varianz) der wahrgenommen Korruption in EL durch die Ausgestaltung liberal-demokratischer Strukturen beschrieben werden kann.

\section{Multiple lineare Regression}

Die Ergebnisse der linearen Einfachregression geben nun Anlass dazu, in einem dritten Schritt eine so genannte multiple lineare Regressionsanalyse durchzuführen. Sie gilt als eine Verallgemeinerung der linearen Einfachregression, mit deren Hilfe der Zusammenhang zwischen einer abhängigen und mehreren unabhängigen Variablen beschrieben werden kann. ${ }^{87}$ Das Ziel der multiplen Regressionsanalyse besteht darin, durch die Einführung weiterer unabhängiger Variablen, die als Determinanten der wahrgenommenen Korruption bereits bestätigt wurden, den tatsächlichen Einfluss liberal-demokratischer Strukturen so exakt wie möglich zu bestimmen. Kann dies gewährleistet werden, spricht man in der Statistik davon, dass die interessierende unabhängige Variable „unter Kontrolle“ ${ }^{88}$ gehalten wird. Das statistische Ergebnis ist demzufolge umso zuverlässiger, desto besser das Kontrollsample ausgewählt wurde. Bei der Beschreibung der Datenerhebung von Korruption in EL wurde unter B.II. bereits ausgeführt, dass das ökonomische Entwicklungsniveau als eine Determinante der wahrgenommenen Korruption in zahlreichen Studien bestätigt wurde. ${ }^{89}$ Sie stellt deshalb für die hier vorliegende Analyse die wichtigste Kontrollvariable dar. ${ }^{90}$ Ferner existiert eine Reihe empirisch bestätigter Faktoren, die Einfluss auf die wahrgenommene Korruption eines Landes ausüben. Eine umfassende Querschnittsanalyse aktuellster Befunde über signifikante und robuste Einflussfaktoren, lieferte Treisman im Jahr

Kohler / Kreuter, (Fn. 82), S. 187.

Kohler / Kreuter, (Fn. 82), S. 207.

89

90

Vgl. La Porta et al.; Ades und di Tella; Treisman, (Fn. 62).

Die Daten für das ökonomische Entwicklungsniveau in EL wurden entnommen von: The World Bank, (Fn. 50). 
2006. ${ }^{91}$ Er konnte darin 90 Prozent der Streuung (Varianz) der wahrgenommenen Korruption erklären.

Nach ausführlichen Analysen der hier vorliegenden Studie konnten davon für EL neben dem ökonomischen Entwicklungsniveau drei Faktoren als statistisch signifikant identifiziert werden. Es wurde festgestellt, dass Länder mit dem ursprünglich aus Großbritannien stammenden „Common Law System“ weniger korrupt sind, als diejenigen mit kontinentaleuropäischen „zivilrechtlichen Systemen“. Die These wird darauf zurückgeführt, dass das „Common Law System“ einen besseren Schutz der Eigentumsrechte vorsieht und die Rechtssprechung insgesamt gewissenhafter durchgeführt wird (effizientere Rechtskultur), wodurch korrupte Handlungen besser kontrolliert werden können. ${ }^{92}$ Ferner konnte bestätigt werden, dass hohe und schwankende Inflation die Kontrolle öffentlicher Ausgaben und Verträge erschwert und dadurch die Korruption erhöht. ${ }^{93}$ Deshalb ist die Währungs- und Preisstabilität in EL eine weitere Determinante der wahrgenommenen Korruption. ${ }^{94}$ Außerdem konnte ein positiver Zusammenhang zwischen Korruption und einer größeren Abschöpfung ökonomischer Renten seitens Bürokraten belegt werden. In diesem Zusammenhang wurde gezeigt, dass dort, wo die Ökonomie mehr vom Export natürlicher Ressourcen abhängig ist, sowohl die Renten als auch die wahrgenommene Korruption tendenziell größer sind. ${ }^{95}$ Aus diesem Grund wird schlussendlich der Kontrollfaktor Anteil an Brennstoffexporten $^{96}$ in das Modell eingeführt. Nach weiteren zahlreichen statistischen Analysen kann damit ein multiples Regressionsmodell berechnet werden, bei dem alle feststellbaren Fehlspezifikationen eliminiert wurden. Damit kann gewährleistet werden, dass die relevante Einflussgröße der liberal-demokratischen Strukturen so exakt wie möglich analysiert wird. Das daraus resultierende multiple Regressionsmodell untersucht den Einfluss liberal-demokratischer Strukturen auf die wahrgenommene Korruption in 81 EL, kontrolliert um die einflussreichsten Determinanten der Preis- und Währungsstabilität, dem Anteil an Brennstoffexporten, dem ökonomischen Entwicklungsniveau und des „Common Law Systems“. Aus diesem Modell wird ersichtlich, dass eine zusätzliche marginale Zunahme liberaldemokratischer Strukturen eine Senkung der wahrgenommenen Korruption um 0,02 Einheiten bewirkt. Dieses Modell erklärt ferner 67 Prozent der Streuung (Varianz) der wahr-

Treisman, (Fn. 62).

La Porta et al., (Fn. 62); Treisman, (Fn. 62). Die Daten für das „Common Law System“ in EL wurden entnommen von: Treisman, (Fn. 62).

Miguel Braun und Rafael di Tella, Inflation, inflation variability and corruption, in: Economics\& Politics 16, 1 (2004), S. 77-100.

94

Die Daten für Preis- und Währungsstabilität in EL wurden entnommen von: Bertelsmann Stiftung, Bertelsmann Transformationsindex 2006, Marktwitschaft-Status Index, http://www.BTI_2006_ Broschuere_D_gesamt.pdf. 
genommenen Korruption in EL und bestätigt einen statistisch hoch signifikanten und robusten Zusammenhang zwischen liberal-demokratischen Strukturen und wahrgenommener Korruption.

Die Stärke der Einflussgröße liberal-demokratischer Strukturen im Vergleich mit den bereits gesicherten Determinanten der wahrgenommenen Korruption kann anhand standardisierter Werte (Beta-Werte) ${ }^{97}$ untersucht werden. Diese Beta-Werte machen deutlich, dass der Einfluss liberal-demokratischer Strukturen $(0,32)$ auf die wahrgenommene Korruption in EL den zweitstärksten Einfluss nach dem ökonomischen Entwicklungsniveau $(0,44)$ ausübt. Der Anteil an Brennstoffexporten $(-0,21)$, die Preis- und Währungsstabilität $(0,19)$ und das „Common Law System“ (-0,19) bleiben auch weiterhin einflussreich. ${ }^{98}$ Auch wenn dieser Einfluss zu keinem eindeutig linearen Zusammenhang führt, so kann festgestellt werden, dass liberal-demokratische Strukturen in einem statistisch signifikanten Zusammenhang mit der wahrgenommenen Korruption in EL stehen.

\section{Schlussfolgerungen}

Auf Basis dieser Ergebnisse soll nun abschließend diskutiert werden, inwiefern liberaldemokratische Strukturen als Hauptbestandteil von GG dazu beitragen, Korruption in EL abzubauen. Zunächst üben liberal-demokratische Strukturen nach dem ökonomischen Entwicklungsniveau den zweitgrößten Einfluss auf die wahrgenommene Korruption aus. Ferner wird in freien EL Korruption weniger wahrgenommen als in teilweise freien EL und dort wiederum weniger als in nicht freien EL. In der Gruppe der nur teilweise freien EL weisen diejenigen, die von FH in die Kategorie Wahldemokratie eingestuft wurden, eine minimal höher wahrgenommene Korruption auf als teilweise freie EL, die von FH nicht in diese Kategorie eingeschlossen wurden. Eine Verbesserung liberal-demokratischer Strukturen hat einen deutlich sichtbaren Einfluss nur in konsolidierten Demokratien.

Folglich können die vorliegenden Befunde die Ergebnisse von Treisman ${ }^{99}$ stützen, dass in defekten Demokratien oder in Autokratien mit einem gewissen Maß an bürgerlichen Freiheiten, kleine Erhöhungen der politischen Rechte keinen konsistenten Einfluss auf die wahrgenommene Korruption haben. Diese Überlegungen über den Einfluss von liberaldemokratischen Strukturen auf die wahrgenommene Korruption in den einzelnen Freiheitskategorien konnten mit den hier verwendeten Analysetechniken jedoch nicht statistisch belegt, sondern nur beobachtet werden. Ferner kann trotz des statistisch signifikanten Zusammenhangs liberal-demokratischer Strukturen auf die wahrgenommene Korruption weder ein perfekt linearer noch ein eindeutiger Richtungszusammenhang konstatiert werden. Wird Korruption durch mehr liberal-demokratische Strukturen reduziert? Oder ist es

97

98

99

Vgl. Backhaus et al., (Fn. 84), S. 61f.

Eigene Berechnungen nach The World Bank und Transparency International, (Fn. 22); Freedom House, (Fn. 65); Bertelsmann Stiftung, (Fn. 94); The World Bank, Fn. (96); Treisman, (Fn. 62).

Treisman, (Fn. 62). 
nicht vielmehr trivial, dass sich die Ausgestaltung liberal-demokratischen Strukturen automatisch verbessert, sofern sich die Korruption verringert?

Diese Frage, die hier leider nicht beantwortet, sondern nur diskutiert werden kann, steht in engem Zusammenhang mit der Problematik der Messung von Korruption und Demokratie. Denn inwiefern lässt sich Korruption überhaupt von Demokratie trennen? Ein erstes Problem bei der Interpretation der hier vorliegenden Ergebnisse resultiert daraus, dass eine Demokratiemessung nach FH bereits die Abwesenheit von Korruption in liberalen Demokratien voraussetzt (Endogenität der unabhängigen Variablen). Dies kann in erster Konsequenz dazu führen, dass der beschriebene Zusammenhang abgeschwächt wird. In zweiter Konsequenz besteht bei der Interpretation der gewonnenen statistischen Ergebnisse die Gefahr, einem tautologischen Fehlschluss zu unterliegen. Das daraus resultierende Endogenitätsproblem der Variablen liberal-demokratische Strukturen scheint in diesem Kontext allerdings nahezu unlösbar. Ebenso kritisch sollte auch die Messung der Korruption aufgrund subjektiver Wahrnehmungen angesehen werden. Auch wenn inzwischen mit dem Globalen Korruptionsbarometer die Reliabilität der Daten des Korruptionswahrnehmungsindex bis zu einem gewissen Grad bestätigt werden konnten, so ist dies bei weitem kein ausreichendes Argument, die Zweifel einer Erhebung an wahrgenommener Korruption auszuräumen, wenngleich sie als etablierte Schätzung in der Forschung längst anerkannt ist.

Doch wie ist nun aufgrund dieser Befunde das Konzept von GG zur Korruptionsminderung zu beurteilen? Die größte Schwierigkeit bei der Bewertung besteht darin, dass die Ausprägung liberal-demokratischer Strukturen in nicht freien und teilweise freien EL nur rudimentär ist und deshalb gemäß der theoretischen Annahmen auch die Kontrollmechanismen nur bedingt in Kraft treten können. Denn tatsächlich wesentlich weniger wahrgenommene Korruption kann, laut der hier vorliegenden Befunde, nur in freien EL beobachtet werden. Dies unterstreicht einerseits die Relevanz der Qualität liberal-demokratischer Strukturen, wie bereits von La Porta et al. ${ }^{100}$ in einer globalen Analyse gezeigt wurde. Andererseits ist diese Problematik sowohl im entwicklungstheoretischen als auch im entwicklungspolitischen Diskurs ein großes Thema, das um die Frage kreist, wie Korruption aufgrund schwach ausgebildeter politischer Institutionen bekämpft werden kann. Der Aufbau starker politischer Institutionen, wie er von Rose-Ackerman ${ }^{101}$ vorgeschlagen wird, klingt zunächst sehr nahe liegend, plausibel und spiegelt sich auch in den empirischen Befunden wider. Doch, wie soll dies in der entwicklungspolitischen Praxis umgesetzt werden, wenn Korruption gerade eben die Möglichkeiten zum Aufbau demokratischer Institutionen verwehrt? Gerade deshalb erscheint es wichtig, die Zusammenhänge zwischen diesen beiden Phänomen besonders in EL weiter zu untersuchen, um adäquate Lösungsansätze zu finden. Viel versprechende Perspektiven eröffnen sich in diesem Zusammenhang beispielsweise durch die rasante Entwicklung und dementsprechend auch der Verbesserung 
der zur Verfügung stehenden Instrumente zur Messung von Korruption und deren Erfassung von nahezu allen Ländern der Welt.

\section{E. Ausblick}

In diesem Aufsatz wurde untersucht, inwiefern liberal-demokratische Strukturen, als Hauptbestandteil des entwicklungspolitischen Konzepts von Good Governance (GG), Korruption in Entwicklungsländern (EL) beeinflussen können. Ausgangspunkt dieser Fragestellung war die inzwischen globale Anwendung des ursprünglich von der Weltbank entwickelten Konzeptes von GG. Es wird inzwischen von allen Geberländern bei der Vergabe ihrer Hilfeleistungen verwendet und steht wegen der Instrumentalisierung von Demokratieförderung zum Zwecke wirtschaftlichen Wachstums in enormer Kritik. In der hier vorliegenden Studie konnte ein statistisch signifikanter und robuster, allerdings nicht-linearer Zusammenhang zwischen liberal-demokratischen Strukturen und wahrgenommener Korruption festgestellt werden. Dabei zeigte sich, dass liberal-demokratische Strukturen neben dem ökonomischen Entwicklungsniveau eine wichtige Determinante der Korruption darstellen. Dieses Ergebnis konnte jedoch nicht bestätigen, dass Demokratieförderung als wesentlicher Bestandteil von GG eindeutig und über alle EL hinweg reduzierend auf das Korruptionsniveau wirkt. Vielmehr wird unter wissenschaftlichen Gesichtpunkten aufgrund der zahlreichen Unsicherheiten in Messung und Analyse empfohlen, gezielte Länderanalysen durchzuführen, um eine optimale Korruptionsminderungsstrategie für das jeweilige betroffene Land $\mathrm{zu}$ entwerfen. Lässt man ferner alle wissenschaftlichen Unsicherheiten außen vor, erscheint es darüber hinaus äußerst schwierig, auf dieses Ergebnis gezielte entwicklungspolitische Handlungsempfehlungen zu gründen. Denn nimmt man an, dass gezielte Demokratieförderung korrupte Strukturen eindämmen kann, so bleibt immer noch die Frage, mit welchen Mitteln viele der EL mit ihren defekten Demokratien oder rudimentären Autokratien eine bessere Regierungsführung vorantreiben sollen. Diese alles entscheidende Frage, die auch die Weltbank nicht beantworten kann, ist in der aktuellen entwicklungspolitischen Diskussion äußerst präsent und wird derzeit beispielsweise vom norwegischen „Anti Corruption Ressource Center (U4)“ des Christian Michelsen Instituts untersucht. Darüber hinaus sollte GG immer nur eine unter mehreren Maßnahmen darstellen, die gezielt in die kulturellen und sozio-ökonomischen Gegebenheiten eines Landes eingebettet sind, um eine effiziente Korruptionsbekämpfung anzusteuern. 